\title{
Effects of canonical color, luminance, and orientation on sustained inattentional blindness for scenes
}

\author{
Kelly Webster ${ }^{1} \cdot$ Jason Clarke ${ }^{2} \cdot$ Arien Mack $^{2} \cdot$ Tony Ro $^{1}$
}

Published online: 9 July 2018

(C) The Psychonomic Society, Inc. 2018

\begin{abstract}
Whether scene gist perception occurs automatically and unconsciously has been the subject of much debate. In addition to demonstrating a new method that adapts the Mack and Rock (1998) inattentional blindness cross procedure to allow for sustained inattentional blindness over a large number of trials, we report evidence from a series of experiments that shows that canonical scene features reduce inattentional blindness to scenes by facilitating the extraction of scene gist. When attentional demands are high, the combination of canonical color, canonical luminance, and canonical orientation reduces rates of inattentional blindness. However, when attentional demands are reduced, canonical features are independently sufficient to facilitate gist extraction and to capture attention. These results demonstrate that canonical color, canonical luminance, and canonical orientation all contribute to scene gist perception, and that when attentional demands are high, only highly canonical stimuli are sufficient to capture attention.
\end{abstract}

Keywords Divided attention $\cdot$ Inattention $\cdot$ Color $\cdot$ Light $\cdot$ Scene perception

A current controversy in the perception literature is whether scene gist can be processed automatically and without attention. Early work has shown that despite their complexity, perception of scenes by the human visual system is swift and highly efficient. Potter's (1975) scene classification experiment, for example, showed that participants are equally adept at recognizing a briefly presented target scene when only a scene name is given as compared to when they have foreknowledge of the exact image of the target, suggesting that scenes are very rapidly processed up to the level of meaning. This earlier finding is supported by numerous other studies demonstrating that scenes can be recognized in a single glance and often as quickly as a component object (Biederman, Mezzanotte, \& Rabinowitz, 1982; Intraub, 1997). This rapid recognition suggests that scenes do not have to be recognized object-by-object, but rather

Kelly Webster

kwebster@gradcenter.cuny.edu

Tony Ro

tro@gc.cuny.edu

1 Program in Psychology, Graduate Center of the City University of New York, New York, NY, USA

2 Department of Psychology, New School for Social Research, New York, NY, USA can be recognized by more global, holistic features. Evidence for this comes from low-level vision research, which suggests that certain low-level features, such as color, luminance, movement, and depth, may play a crucial role in facilitating rapid recognition (Livingstone \& Hubel, 1987).

Much of the current literature on scene perception has investigated the ability to recognize and identify scenes under conditions of full attention while impairing perception in a variety of ways, including short presentation times and manipulation of image features. For example, scenes can be degraded using spatial frequency filters, rendering typically complex scenes into amorphous blobs. Nevertheless, numerous studies have demonstrated that in such scenarios, luminance information is sufficient to support recognition of the degraded scenes, and this recognition occurs even without recognition of component objects (Oliva \& Schyns, 1997; Schyns \& Oliva, 1994).

Whether color additionally contributes to the efficient perception of scenes is unclear. A body of work examining whether pictures of objects match a previously displayed lexical name or category indicate that color has no effect on correct verification (e.g., Biederman \& Ju, 1988; Davidoff \& Ostergaard, 1988). Similarly, studies examining categorization of scenes have shown no benefit of color on correct categorization (Delorme, Richard, $\&$ Fabre-Thorpe, 2000). Other studies on scene recognition have indicated that there are conditions in which color provides a 
distinct advantage in scene perception. When scenes are degraded through spatial frequency filters, eliminating the ability to recognize component objects, scenes are recognized more efficiently when accurate color information is provided in addition to luminance information (Oliva \& Schyns, 2000). Similarly, Castelhano and Henderson (2008) showed that color aided the activation of scene gist when scenes were degraded by blurring. Under these same conditions, inverted color interfered with the activation of scene gist more than luminance-only information. Furthermore, when testing familiarity with previously presented scenes, participants performed better with colored images than with grayscale, but only if the scene color information was accurate (Wichmann, Sharpe, \& Gegenfurtner, 2002; although Nijboer, Kanai, de Haan, \& van der Smagt, 2008, found increased false alarms to colored natural scenes in a memory recognition task, likely because color led to faster identification of the gist and less scrutiny of the scenes). Therefore, it is only the addition of accurate color information, which we will refer to as canonical color, that facilitates the activation of scene gist.

However, color information is not equally important for all scenes. Colors that are informative of scene content, such as blue indicating the presence of sky or water or green indicating foliage, facilitate recognition. Scenes in which color is informative will be recognized and identified faster if color information is provided than when only luminance information is provided. However, when color is not informative of scene content, the addition of color information does not improve recognition (Goffaux et al., 2005; Oliva \& Schyns, 2000).

Although the efficiency of scene perception under conditions of full attention has been well-demonstrated, the capacity of scene perception under constrained attention is less clear. When attention is divided between multiple tasks, participants are nevertheless adept at scene recognition (Li, VanRullen, Koch, \& Perona, 2002; Rousselet, Fabre-Thorpe, \& Thorpe, 2002; however, see Evans \& Treisman, 2005, who demonstrated that although detection is high under these conditions, identification is considerably lower). Yet, when inattentional blindness is induced by directing attention toward a single primary task, perception of unexpected scenes is severely impaired (Cohen, Alvarez, \& Nakayama, 2011; Mack \& Clarke, 2012). Despite the demonstrated importance of low-level features-including luminance and color - in facilitating scene recognition under conditions of full attention, the role and relative importance of these features in facilitating scene perception and attention capture under conditions of reduced attention remains an understudied area.

In the present study, we investigated the effects of color, luminance, and orientation on inattentional blindness to scenes. Specifically, we investigated whether features that were informative of scene gist (i.e., canonical color, luminance, and orientation) promote gist extraction under conditions of reduced attention and ultimately facilitate attention capture. Furthermore, we investigated whether canonical color can enhance gist extraction relative to canonical luminance, as was previously shown under conditions of full attention (Castelhano \& Henderson, 2008; Oliva \& Schyns, 2000; Wichmann et al., 2002). We investigated the role of color and luminance by using identical scenes rendered into four conditions: canonical color, grayscale (canonical luminance), RGB color-inverted (non-canonical color and non-canonical luminance), and $L^{*} a * b *$ color-inverted (canonical luminance and non-canonical color). We hypothesized that consistency with typical scene characteristics may facilitate recognition and detection of canonically featured scenes relative to manipulated scenes. To further test this, we included a fifth condition designed to impair gist extraction from the scenes without altering the colors or luminance. The fifth condition consisted of scenes that had canonical color and luminance but were orientationinverted (upside-down). Shore and Klein (2000) demonstrated that inverting scene orientation eliminated the center-of-interest effect for detecting gist-related changes, suggesting that this is an effective method of impairing gist extraction.

To better elucidate the effects of these factors on inattentional blindness, we extended a sustained inattentional blindness paradigm reported by Mack and Clarke (2012), since rates of inattentional blindness in single critical trial paradigms tend to be high and might obscure differences between conditions. To sustain inattentional blindness over many trials, we presented 20 scenes three times each (Exp. 1) or 60 scenes one time each (Exp. 2) and measured awareness of the scenes at the conclusion of each experiment. Reduced inattentional blindness to colored scenes (canonically colored, RGB color-inverted, $L * a * b *$ color-inverted, and orientation-inverted) relative to grayscale scenes would suggest that color affects inattentional blindness by adding additional contrast information that can be used to parse out scene elements. Reduced inattentional blindness to only canonically colored scenes would suggest that color affects inattentional blindness through a higher-level mechanism in which scene color must be consistent with typical color relationships. Similarly, demonstrating an advantage for $L * a * b *$ colorinverted scenes relative to RGB color-inverted scenes and an advantage of canonically oriented scenes relative to orientation-inverted scenes would demonstrate that luminance and orientation, respectively, also affect inattentional blindness through a similar higher-level mechanism.

\section{Experiment 1: Inattentional blindness to scenes under dual-task conditions}

\section{Method}

This research conformed to the tenets of the Declaration of Helsinki and was approved by the Institutional Review Board of the City University of New York. In Experiment 1, we examined the effects of canonical features on inattentional blindness 
using a between-subjects design with five conditions. Each condition used the same set of 20 scenes, which were rendered into four conditions in which color and luminance were manipulated: canonically colored, grayscale, RGB color-inverted, and $L^{*} a^{*} b^{*}$ color-inverted. We expected that canonical coloring would facilitate scene gist perception and thus reduce inattentional blindness. In a fifth condition, we examined whether extraction of scene meaning could be impaired without altering scene color or luminance by inverting the orientation of the scenes. We anticipated that inverting the orientation of canonically colored scenes would interfere with the efficient processing of colored scenes, producing increased inattentional blindness.

Participants A total of 180 participants (124 females, 56 males; mean age of 22.28 years, $18-53$ years old) completed this study for course credit or monetary compensation. The sample size was determined in concordance with similar studies of inattentional blindness (e.g., Clarke, Ro, \& Mack, 2013; Mack $\&$ Clarke, 2012). Of the 180 participants, 36 completed the experiment viewing canonically colored scenes (canonical color condition), 36 completed the experiment viewing grayscale scenes (grayscale condition), 36 completed the experiment viewing RGB color-inverted scenes (RGB color-inverted condition), 36 completed the experiment viewing $L^{*} a^{*} b^{*}$ colorinverted scenes $\left(L^{*} a^{*} b^{*}\right.$ color-inverted condition), and $36 \mathrm{com}$ pleted the experiment viewing orientation-inverted scenes (orientation-inverted condition). All participants gave written informed consent and had normal or corrected-to-normal vision.

Stimuli and procedure All stimuli were presented against a white background on a CRT monitor running at a $100-\mathrm{Hz}$ refresh rate using custom software written in Visual C++ with Microsoft DirectX libraries. The stimuli were created first in canonical color. Twenty scenes were selected from Google image archives to be used as the critical stimuli. Frequency-scrambled scenes were generated by taking the Fourier transform of each color channel and independently phase-scrambling the channels. Three variations of a random-dot mask were created that varied only in the placement of the dots. An example of this mask can be seen in Fig. 1. The random-dot masks were originally created for the canonical color condition by randomly generating RGB values for the dots at different locations. The grayscale, RGB color-inverted, $L^{*} a * b *$ color-inverted, and orientation-inverted versions of scenes and the frequency-scrambled scenes were created from the canonically colored stimuli. Likewise, the grayscale, RGB color-inverted, $L * a * b *$ color-inverted, and orientation-inverted masks were created from the original random-dot masks. The grayscale images were created by removing all color while retaining the luminance differences in the stimuli. Color inversion was achieved by taking the negative of all color channels in RGB color space and by taking the inversion of the $a^{*}$ and $b^{*}$ channels in $L^{*} a^{*} b^{*}$ color space for the RGB and $L^{*} a^{*} b^{*}$ color-inverted conditions, respectively. Orientation inversion was achieved by rotating the canonically colored images by 180 degrees.

A modified version of the Mack and Rock (1998) cross task was used to induce inattentional blindness. Participants completed 18 practice trials, followed by 100 trials of the inattentional blindness task. Each trial began with presentation of a fixation square (subtending $0.1^{\circ} \times 0.1^{\circ}$ of visual angle) for $1,000 \mathrm{~ms}$ (Fig. 1). An image (subtending $5.5^{\circ} \times 4.2^{\circ}$ of visual angle) was then presented at fixation while a cross (from $2.6^{\circ}$ to $5.8^{\circ}$ of visual angle for each of the horizontal and vertical components) was presented in the upper left, upper right, lower left, or lower right quadrant of the screen $\left(3.2^{\circ}\right.$ of visual angle from fixation). The quadrant of cross presentation was randomly selected on each trial. The cross and the scene or scrambled scene stimuli remained on the screen for $100 \mathrm{~ms}$. On 60 trials (critical trials), the image presented at fixation was a randomly selected scene out of the pool of 20 scenes; each scene was repeated three times over the duration of the experiment. On the remaining 40 trials (non-critical trials), the image presented at fixation was a frequency-scrambled scene. The

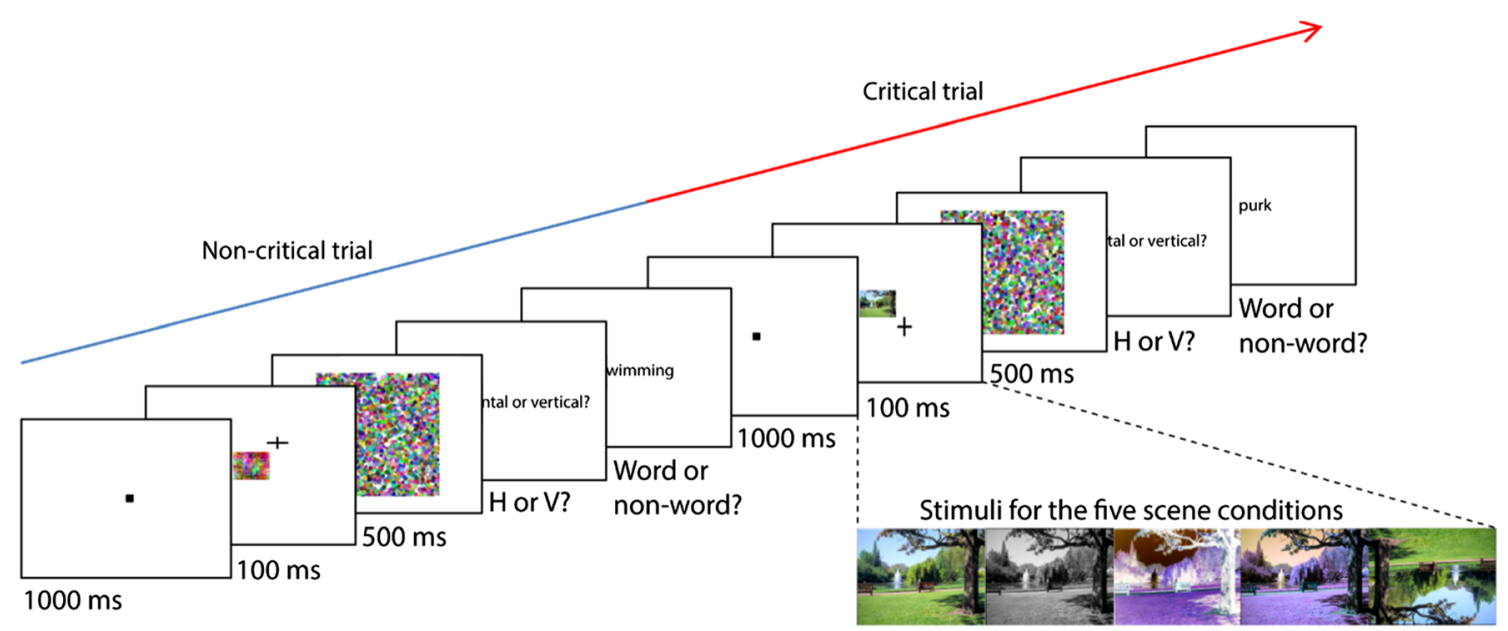

Fig. 1 Stimuli and experimental design for Experiment 1. The inset shows example scene stimuli for the canonical color condition, grayscale condition, RGB color-inverted condition, $L * a * b *$ color-inverted condition, and orientation-inverted condition, respectively 
practice trials and the first ten trials of the experiment were always non-critical trials, after which the two trial types were randomly intermixed. After the presentation of the image and cross, one of three randomly selected dot masks (subtending $18.3^{\circ} \times 18.3^{\circ}$ visual angle) was presented on the screen for $500 \mathrm{~ms}$. Participants were instructed to report the longer arm of the cross using the mouse. Participants pressed the left mouse button if they thought the horizontal line was longer, and the right mouse button if they thought the vertical line was longer. A maximum of 2,000 ms was allotted for making this response.

Following the cross response, a letter string was presented on the screen. The letter string was either a related word, a non-related word, or a non-word. Each scene was followed by one of these three letter strings an equal number of times. Participants were instructed to report whether the string formed a word or a nonword. The accuracy and reaction time on this lexical decision task were used in an attempt to measure lexical priming. However, because the primes and probes were in different formats (i.e., image vs. word) and the primes were presented long (up to 2,600-ms SOA) before the probes, we were unable to find consistent evidence of priming in this experiment (cf. Branigan, Pickering, \& Cleland, 1999; Kiefer \& Spitzer, 2000). ${ }^{1}$ Therefore, the priming manipulations and analyses will not be discussed further.

At the end of the experiment, awareness of the scenes was assessed through three question prompts. First, we assessed scene awareness broadly by asking participants whether they had noticed anything on the screen aside from the cross and mask pattern. This was followed by a more direct question: "Did you notice any scenes?" Finally, participants were told that scenes had been presented during the experiment and they were asked to describe any that they could recall. Participants who reported awareness of the scenes after any of the three prompts were considered non-inattentionally blind. Participants who did not report being able to see any of the scenes were considered to have been inattentionally blind.

\section{Results}

Inattentional blindness results Eleven percent (4 participants) of the participants reported that they were not aware of the canonically colored scenes, $42 \%$ (15 participants) of participants reported that they were not aware of the grayscale scenes, $47 \%$ (17 participants) of participants reported that they were not aware of the RGB color-inverted scenes, $31 \%$ (11 participants) of participants reported that they were not aware of the $L * a * b *$ colorinverted scenes, and $31 \%$ (11 participants) of participants reported that they were not aware of the orientation-inverted scenes (see Fig. 2). There was a significant difference $\left[\chi^{2}(4, N=180)=\right.$ 12.62, $p=.01, V=.26]$ among the rates of inattentional blindness

\footnotetext{
${ }^{1}$ In a control experiment that used a shorter SOA between the centrally presented image primes and word probes $(600 \mathrm{~ms})$, no crosshairs, and no line judgment task, priming was also not reliably measured with these stimuli.
}

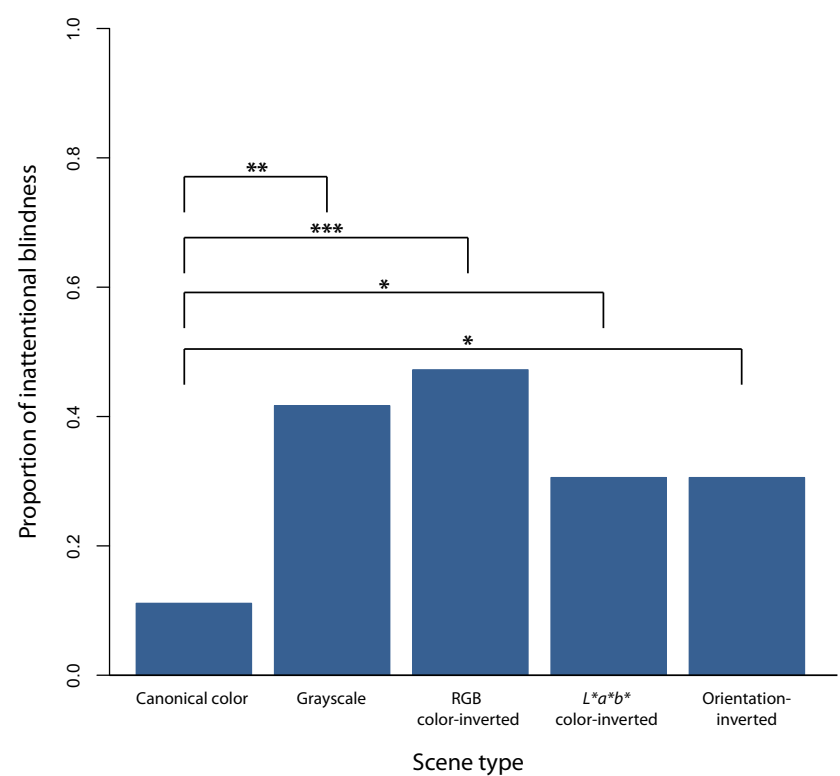

Fig. 2 Proportions of inattentionally blind participants by scene type. Inattentional blindness was significantly reduced for canonically colored scenes as compared to grayscale, RGB color-inverted, $L^{*} a^{*} b^{*}$ color-inverted, and canonically colored but orientation-inverted scenes. ${ }^{*} p<.05, \stackrel{* *}{p} p<.01,{ }^{* * * *} p<.001$.

for the five conditions. The significant $\chi^{2}$ test was followed up by planned pairwise comparisons. Inattentional blindness to canonically colored scenes was significantly reduced as compared to grayscale $\left[\chi^{2}(1, N=72)=8.65, p=.003, V=.35\right]$, RGB colorinverted $\left[\chi^{2}(1, N=72)=11.36, p=.001, V=.40\right], L^{*} a^{*} b^{*}$ colorinverted $\left[\chi^{2}(1, N=72)=4.13, p=.04, V=.24\right]$, and orientationinverted $\left[\chi^{2}(1, N=72)=4.13, p=.04, V=.24, p_{\text {augmented }}=[.062\right.$ $\left..065]^{2}\right]$ scenes. None of the other comparisons were significant (all $\chi^{2} \mathrm{~s} \leq 2.10$, all $p \mathrm{~s} \geq .15$ ).

\footnotetext{
${ }^{2}$ Initially, this dataset was analyzed on 120 participants with uneven sample sizes across the conditions. To ensure that the inattentional blindness effect was not driven by sample size differences in some of the conditions, we recruited additional participants in each condition to make the sample sizes equal. All of the significant effects reported here were significant in the original dataset, with the exception of the comparison between canonically colored scenes and orientation-inverted scenes, which only approached significance in the original dataset. To determine whether augmenting the sample size affected the Type I error rate, we used the $p_{\text {augmented }}$ statistic, described in Sagarin, Ambler, and Lee (2014), which signifies the Type I error inflation resulting from post-hoc data augmentation. This statistic is based on the original and augmented sample sizes, the critical value for statistical significance, the $p$ value in the final (augmented) dataset, and the maximum $p$ value $\left(p_{\max }\right)$ from the original dataset that would cause the researcher to augment the sample size. The resulting statistic, $p_{\text {augmented, }}$ is a range that represents the Type I error inflation resulting from augmenting the dataset. The low end of the range represents the inflation in Type I error under the most conservative augmentation scenario, in which the value for $p_{\max }$ was equal to the original $p$ value for the comparison. The upper end of the range represents the inflation in Type I error under the least conservative augmentation scenario, in which $p_{\max }=1$. As a result of this calculation, $p_{\text {augmented }}$ will always be greater than the critical value (in this case, .05). In this study, for comparisons in which the $p$ value was significant in the original analyses, post-hoc data augmentation had a negligible effect on Type I error rates. For the comparison between canonically colored scenes and canonically colored but orientation-inverted scenes, which approached significance in the original analyses, the increase in Type I error was small, with $p_{\text {augmented }}=[.062 .065]$. We believe this justifies the use of the full sample.
} 
Cross task results Trials were included in the cross task analysis only if a response was made to the cross task. After trial exclusion, $96 \%$ of the trials were included in the analysis. Percent correct performance rates on the cross task were submitted to a repeated measures analysis of variance (ANOVA) with subjects as a random-effects factor, trial type as a within-subjects factor, and condition and inattentional blindness status as between-subjects factors.

We observed no significant difference in cross task performance for scene trials as compared to frequencyscrambled scene trials (see Table 1), $F(1,170)=1.36, p$ $=.25, \eta_{\mathrm{p}}{ }^{2}=.01$. There was also no significant difference in cross task performance between inattentionally blind and non-inattentionally blind participants, $F(1,170)=$ $0.22, p=.64, \eta_{\mathrm{p}}{ }^{2}=.001$. However, we did find a significant difference in cross task performance across the five conditions, $F(4,170)=3.46, p=.009, \eta_{\mathrm{p}}{ }^{2}=.08$. Planned pairwise comparisons using Bonferroni corrections for multiple comparisons revealed that the performance difference was due to significantly reduced cross task performance among those who saw grayscale images as compared to those who saw RGB color-inverted images, $t(70)$ $=3.03, p=.03, d=.71$. There were no other significant differences in cross performance between the other conditions (all $t \mathrm{~s} \leq 2.78$, all $p \mathrm{~s} \geq .07$ ), nor were there any significant interactions (all $F_{\mathrm{s}} \leq 1.65$, all $p \mathrm{~s} \geq .20$ ).

\section{Experiment 2: Inattentional blindness to scenes under single task conditions}

\section{Method}

Inclusion of the priming task in Experiment 1 may have influenced the inattentional blindness results in two ways. First, presentation of a word that was related to the scene preceding it or to a scene that would follow it some trials later may have triggered perception of the scene, either through retroperception (Sergent et al., 2013) or priming. Furthermore, repeated presentations of the same scenes may have aided in the perception and recognition of later presentations of those scenes (Bar \& Biederman, 1998; Bartram, 1974; Biederman \& Cooper, 1991; Haber \& Hershenson, 1965; Schacter, Delaney, \& Merikle, 1990). To rule out these effects, we conducted a control experiment on Amazon Mechanical Turk in which all of the priming aspects of Experiment 1 were removed, thus simplifying the task from a dual to a single one.

Participants A total of 360 participants (287 males, 73 females; mean age $=33.01,18-80$ years) completed this experiment for monetary compensation. Of the 360 participants, 72 completed the experiment viewing canonically colored stimuli, 72 completed the experiment viewing grayscale stimuli, 72 completed the experiment viewing RGB color-inverted stimuli, 72 completed the experiment viewing $L * a * b *$ color-

Table 1 Proportions of correct responses (with standard errors) on the cross task in Experiment 1

\begin{tabular}{|c|c|c|c|c|}
\hline Scene Type & Participant Status & Scene Trials & Nonscene Trials & Total \\
\hline \multirow[t]{3}{*}{ Canonical color } & Inattentionally blind & $.64(.06)$ & $.57(.10)$ & $.61(.09)$ \\
\hline & Non-inattentionally blind & $.70(.02)$ & $.69(.02)$ & $.70(.03)$ \\
\hline & Total & $.70(.02)$ & $.68(.02)$ & $.65(.05)$ \\
\hline \multirow[t]{3}{*}{ Grayscale } & Inattentionally blind & $.60(.06)$ & $.57(.05)$ & $.59(.05)$ \\
\hline & Non-inattentionally blind & $.56(.05)$ & $.59(.06)$ & $.57(.04)$ \\
\hline & Total & $.58(.04)$ & $.58(.04)$ & $.58(.03)$ \\
\hline \multirow[t]{3}{*}{ RGB color-inverted } & Inattentionally blind & $.77(.03)$ & $.76(.03)$ & $.77(.04)$ \\
\hline & Non-inattentionally blind & $.69(.04)$ & $.66(.05)$ & $.68(.04)$ \\
\hline & Total & $.73(.03)$ & $.71(.03)$ & $.72(.03)$ \\
\hline \multirow[t]{3}{*}{$L * a * b *$ color-inverted } & Inattentionally blind & $.66(.04)$ & $.67(.06)$ & $.66(.05)$ \\
\hline & Non-inattentionally blind & $.72(.02)$ & $.72(.03)$ & $.72(.04)$ \\
\hline & Total & $.70(.02)$ & $.70(.03)$ & $.69(.03)$ \\
\hline \multirow[t]{3}{*}{ Orientation-inverted } & Inattentionally blind & $.67(.06)$ & $.68(.07)$ & $.68(.05)$ \\
\hline & Non-inattentionally blind & $.69(.03)$ & $.71(.03)$ & $.70(.04)$ \\
\hline & Total & $.68(.03)$ & $.69(.04)$ & $.69(.03)$ \\
\hline \multirow[t]{3}{*}{ Total } & Inattentionally blind & $.67(.03)$ & $.64(.03)$ & $.66(.03)$ \\
\hline & Non-inattentionally blind & $.66(.02)$ & $.66(.02)$ & $.64(.02)$ \\
\hline & Total & $.67(.02)$ & $.65(.02)$ & $.66(.02)$ \\
\hline
\end{tabular}


inverted stimuli, and 72 completed the experiment viewing orientation-inverted stimuli. All participants gave online informed consent, approved by the Institutional Review Board of the City University of New York.

Stimuli and procedure The stimuli and procedure of this experiment matched those for Experiment 1, with the following exceptions. Sixty unique scenes were selected from Google image archives to be used as the critical stimuli. Of the 60 scenes, 17 were identical to ones used in Experiment 1, three were improved versions from Experiment 1, and the remaining 40 were previously unused scenes. Because this experiment was conducted remotely via Amazon Mechanical Turk, the stimulus sizes could not be precisely controlled and thus were estimated post-hoc. Participants were instructed to sit at arm's length from their computer monitor during the experiment. At the end of the experiment, participants measured a standard stimulus (the longer side of a credit card) against a gauge of a set number of pixels that was drawn on the screen. This allowed us to convert between physical size and pixels for each participant. The visual angles of the presented stimuli were computed from these values and averaged across participants. ${ }^{3}$ However, because compliance with the instructions could not be ensured, the visual angles presented here are estimates and may not have been accurate for all participants.

Participants completed 18 practice trials, followed by 100 trials of the inattentional blindness task. In each condition, the trial began with the presentation of a fixation square (subtending on average $0.10^{\circ} \times 0.10^{\circ}$ of visual angle) for $1,000 \mathrm{~ms}$ (Fig. 3). An image (subtending on average $5.13^{\circ} \times 3.91^{\circ}$ of visual angle) was then presented at fixation while a cross (subtending on average $2.40^{\circ}$ to $5.48^{\circ}$ of visual angle for each of the horizontal and vertical components) was presented in the periphery (on average $2.97^{\circ}$ of visual angle from fixation). These stimuli remained on the screen for $100 \mathrm{~ms}$. On 60 trials (critical trials), the image presented at fixation was randomly selected from a pool of 60 scenes; each scene was presented once. On the remaining 40 trials (non-critical trials), the image presented at fixation was a frequency-scrambled scene. The practice trials and the first ten trials of the experiment were always noncritical trials, after which the two trial types were randomly intermixed. Participants were instructed to report the longer

\footnotetext{
${ }^{3}$ Seventeen of the participants either did not report a standard stimulus measurement or reported an incomplete/incorrect measurement (e.g., they measured the standard stimulus against something other than the gauge drawn on the screen). The data from these participants were not included in any of the analyses of stimulus size, but were included in the task analyses. Because participants did not report arm length, which is necessary to compute visual angle, average arm length was used in visual angle calculations. Average arm length was estimated on the basis of previously studied relationships between arm length and height, using the formula .44* average height (Chaffin, Andersson, \& Martin, 1999). Average height of American adults was derived from Fryar, Gu, Ogden, and Flegal (2016). Because calculations of visual angle are based on averages, rather than reported measurements, the visual angle values reported here serve as rough estimates of stimulus size.
}

arm of the cross using the keyboard. Participants pressed the " $\mathrm{B}$ " key if they thought the horizontal line was longer, and the "M" key if they thought the vertical line was longer. A maximum of 2,000 ms was allotted for making this response.

At the end of the experiment, awareness of the scenes was assessed through three question prompts. First, we assessed scene awareness broadly by asking participants whether they had noticed anything on the screen aside from the cross and mask pattern. This was followed by a more direct question: "Did you notice any scenes?" Finally, participants were told that scenes had been presented during the experiment and were asked to describe any that they could recall. Participants who reported seeing some of the scenes on any of the three questions were considered non-inattentionally blind. Participants who did not report being able to see any of the scenes were considered to have been inattentionally blind.

\section{Results}

Inattentional blindness results Twenty-two percent (16 participants) of the participants reported that they were not aware of the canonically colored scenes, 22\% (16 participants) of participants reported that they were not aware of the grayscale scenes, $44 \%$ (32 participants) of participants reported that they were not aware of the RGB color-inverted scenes, 24\% (17 participants) of participants reported that they were not aware of the $L^{*} a^{*} b^{*}$ color-inverted scenes, and 29\% (21 participants) of participants reported that they were not aware of the orientation-inverted scenes (see Fig. 4).

We found a significant difference $\left[\chi^{2}(4, N=360)=12.67\right.$, $p=.01, V=.19]$ in the rates of inattentional blindness across the five conditions. The significant $\chi^{2}$ test was followed up by planned pairwise comparisons. The overall significant $\chi^{2}$ test was driven largely by significantly increased rates of inattentional blindness to RGB color-inverted scenes as compared to the canonically colored scenes $\left[\chi^{2}(2, N=144)=\right.$ $8.00, p=.005, V=.24]$, grayscale scenes $\left[\chi^{2}(2, N=144)=\right.$ $8.00, p=.005, V=.24]$, and $L^{*} a * b^{*}$ color-inverted scenes $\left[\chi^{2}(2, N=144)=6.96, p=.008, V=.22\right]$. The rate of inattentional blindness to RGB color-inverted scenes was also marginally increased relative to the rate of inattentional blindness to orientation-inverted scenes $\left[\chi^{2}(2, N=144)=3.61, p=\right.$ $.06, V=.16]$. None of the other comparisons between conditions were significant (all $\chi^{2} \mathrm{~s} \leq .91$, all $p \mathrm{~s} \geq .34$ ).

Cross task results Trials were included in the cross task analysis only if a response was made to the cross task. After trial exclusion, $98 \%$ of the trials were included in the analysis. The percent correct performance rates on the cross task were submitted to a repeated measures ANOVA with subjects as a random-effects factor, trial type as a within-subjects factor, and condition and inattentional blindness status as betweensubjects factors. 


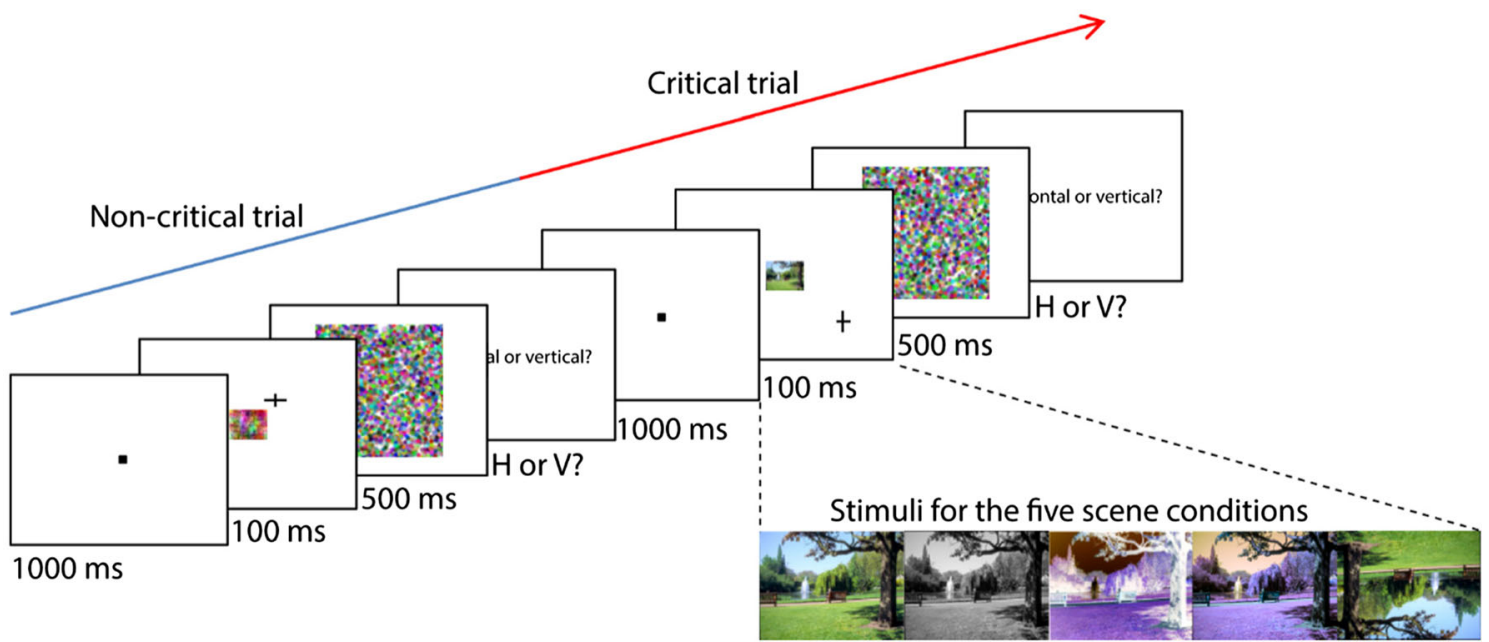

Fig. 3 Stimuli and experimental design for Experiment 2. The inset shows example scene stimuli for the canonical color condition, grayscale condition, RGB color-inverted condition, $L * a * b *$ color-inverted condition, and orientation-inverted condition, respectively

There was no significant difference in cross task performance for scene trials versus frequency-scrambled scene trials (see Table 2), $F(1,350)=0.68, p=.41, \eta_{\mathrm{p}}^{2}=.002$. We also observed no significant difference in cross task performance between inattentionally blind and non-inattentionally blind participants, $F(1,350)=0.004, p=.89, \eta_{\mathrm{p}}^{2}<.001$, and no significant difference in cross task performance between the five experimental conditions, $F(4,350)=.28, p=.89, \eta_{\mathrm{p}}{ }^{2}=$ .003 . Furthermore, there were no significant interactions (all $F_{\mathrm{s}} \leq 1.41$, all $p \mathrm{~s} \geq .23$ ).

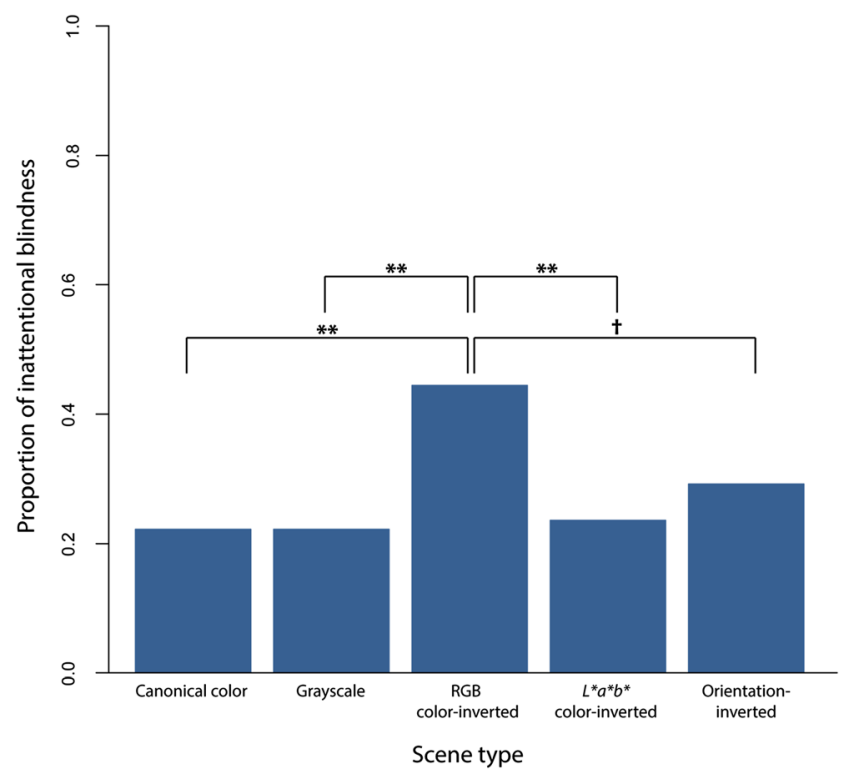

Fig. 4 Proportions of inattentionally blind participants by condition in Experiment 2. Inattentional blindness was significantly increased for RGB color-inverted scenes relative to the canonically colored scenes, grayscale scenes, and $L * a * b *$ color-inverted scenes; this increase was only marginally significant relative to the orientation-inverted scenes. ${ }^{\dagger} p$ $<.1,{ }^{* *} p<.01$.
Comparison of Experiments 1 and 2: Inattentional blindness results The inattentional blindness rates were submitted to a repeated measures ANOVA with subjects as a random-effects factor and condition and experiment as between-subjects factors. We found no significant difference in the overall rates of inattentional blindness in Experiments 1 and 2, $\chi^{2}(1, N=540)$ $=0.87, p=.35, V=.04$. To determine whether the patterns of inattentional blindness results differed across the two experiments, we performed planned pairwise comparisons across the different scene conditions. There were no significant differences in rates of inattentional blindness to canonically colored scenes $\left[\chi^{2}(2, N=108)=1.96, p=.16, V=.13\right]$, RGB color-inverted scenes $\left[\chi^{2}(2, N=108)=0.07, p=.78, V=.03\right]$, $L * a * b *$ color-inverted scenes $\left[\chi^{2}(2, N=108)=0.60, p=.44\right.$, $V=.07]$, or orientation-inverted scenes $\left[\chi^{2}(2, N=108)=0.02\right.$, $p=.88, V=.01]$ across the two experiments. However, inattentional blindness to the grayscale scenes was significantly reduced in Experiment 2 as compared to Experiment 1 $\left[\chi^{2}(2, N=108)=4.43, p=.04, V=.20\right]$.

Comparison of Experiments 1 and 2: Cross task results To determine whether the overall task in Experiment 2 was easier, either because the priming component of the experiment was removed or because the size of the stimuli may have been larger on some monitors, we compared the cross task performance between Experiments 1 and 2. As before, trials were included in the cross task analysis only if a response was made to the cross task. After trial exclusion, $97.64 \%$ of trials were included in the analysis. The percent correct performance rates on the cross task were submitted to a repeated measures ANOVA with subjects as a random-effects factor, trial type as a within-subjects factor, and condition, inattentional blindness status, and experiment as between-subjects factors.

A significant difference in performance on the cross task was apparent between the two experiments, $F(1$, 
Table 2 Proportions of correct responses (with standard errors) on the cross task in Experiment 2

\begin{tabular}{|c|c|c|c|c|}
\hline Scene Type & Participant Status & Scene Trials & Nonscene Trials & Total \\
\hline \multirow[t]{3}{*}{ Canonical color } & Inattentionally blind & $.74(.04)$ & $.74(.04)$ & $.74(.04)$ \\
\hline & Non-inattentionally blind & $.75(.02)$ & $.76(.02)$ & $.75(.04)$ \\
\hline & Total & $.75(.02)$ & $.75(.02)$ & $.75(.02)$ \\
\hline \multirow[t]{3}{*}{ Grayscale } & Inattentionally blind & $.71(.05)$ & $.75(.05)$ & $.73(.04)$ \\
\hline & Non-inattentionally blind & $.74(.02)$ & $.76(.02)$ & $.75(.02)$ \\
\hline & Total & $.73(.02)$ & $.75(.02)$ & $.74(.02)$ \\
\hline \multirow[t]{3}{*}{ RGB color-inverted } & Inattentionally blind & $.77(.02)$ & $.76(.02)$ & $.77(.03)$ \\
\hline & Non-inattentionally blind & $.75(.02)$ & $.75(.02)$ & $.75(.02)$ \\
\hline & Total & $.76(.02)$ & $.76(.02)$ & $.76(.02)$ \\
\hline \multirow[t]{3}{*}{$L * a * b *$ color-inverted } & Inattentionally blind & $.76(.03)$ & $.77(.03)$ & $.76(.04)$ \\
\hline & Non-inattentionally blind & $.74(.02)$ & $.74(.02)$ & $.74(.02)$ \\
\hline & Total & $.75(.02)$ & $.75(.02)$ & $.75(.02)$ \\
\hline \multirow[t]{3}{*}{ Orientation-inverted } & Inattentionally blind & $.74(.04)$ & $.73(.04)$ & $.73(.03)$ \\
\hline & Non-inattentionally blind & $.75(.02)$ & $.73(.02)$ & $.74(.02)$ \\
\hline & Total & $.74(.02)$ & $.73(.02)$ & $.74(.02)$ \\
\hline \multirow[t]{3}{*}{ Total } & Inattentionally blind & $.75(.02)$ & $.75(.02)$ & $.75(.02)$ \\
\hline & Non-inattentionally blind & $.74(.01)$ & $.75(.01)$ & $.75(.01)$ \\
\hline & Total & $.75(.01)$ & $.75(.01)$ & $.75(.01)$ \\
\hline
\end{tabular}

$520)=23.06, p<.001, \eta_{\mathrm{p}}{ }^{2}=.04$, with the participants in Experiment 2, without the priming task, performing significantly better $(M=.75, S E=.01)$ than the participants in Experiment $1(M=.67, S E=.01)$. There was also a significant difference in performance on the cross task across the five conditions, $F(4,520)=3.30, p=.01$, $\eta_{\mathrm{p}}{ }^{2}=.03$; however, none of the pairwise comparisons survived Bonferroni corrections for multiple comparisons (all $t \mathrm{~s} \leq 2.32$, all $p \mathrm{~s} \geq .21$ ). We also observed a marginally significant interaction between experiment and condition, $F(4,520)=2.08, p=.08, \eta_{\mathrm{p}}^{2}=.02$. Pairwise comparisons using Bonferroni corrections for multiple comparisons revealed that the performance difference was driven by significantly reduced performance on the cross task in Experiment 1 when participants viewed grayscale scenes relative to Experiment 2, $t(106)=$ $6.00, p<.001, d=.82$. There was no significant difference in performance on the cross task between participants who were inattentionally blind and those who were non-inattentionally blind $\left[F(1,520)=0.21, p=.64, \eta_{\mathrm{p}}{ }^{2}<\right.$ $.001]$ or between trials in which a scene was presented versus non-scene trials $\left[F(1,520)=0.39, p=.54, \eta_{\mathrm{p}}{ }^{2}=\right.$ $.001]$. None of the other interactions were significant (all $F \mathrm{~s} \leq 2.26, p \mathrm{~s} \geq .12$ ).

Performance differences: Load or size? The improved cross task performance in Experiment 2 suggests that this task was easier than in Experiment 1. This difference may have been a consequence of reduced load in Experiment 2 because the lexical decision task was removed, making it easier for participants to concentrate their attention on the cross task. Alternatively, it is possible that the stimuli appeared larger on some monitors in Experiment 2, making this task easier to perform because the differences in cross arm length were larger. Furthermore, this increase in stimulus sizes could have reduced inattentional blindness because the scenes were bigger and more salient.

To assess whether load or stimulus size differences resulted in the differences in performance between Experiments 1 and 2, we took advantage of the fact that some of the participants had larger monitors, and thus larger stimulus sizes, than others in Experiment 2. We estimated the sizes of the stimuli for each participant based on their reported standard stimulus measurements and analyzed the relationship between stimulus size and performance on the cross and inattentional blindness tasks in Experiment 2. There was no significant correlation between standard stimulus measurement and cross task performance, $r(341)=-.03, p=.53$. There was also no significant difference in stimulus size between the participants who were either inattentionally blind $\left(M_{\text {meas. }}=58.49\right.$, range $\left.=30-100\right)$ or noninattentionally blind $\left(M_{\text {meas. }}=54.96\right.$, range $\left.=19-100\right), t(341)$ $=1.77, p=.08$. These results suggest that stimulus size did not significantly affect inattentional blindness rates or cross task performance in Experiment 2, and that any differences found between Experiments 1 and 2 were likely due to differences in load rather than stimulus size. 


\section{Experiment 3: Manipulation check}

We hypothesized that perceiving the scene gist, or the central meaning of a scene, might facilitate conscious awareness of scenes. Although the scene gist was identical across the different manipulations in the two experiments, we anticipated that the color, luminance, and orientation manipulations would affect how readily the gist could be extracted, and thus how readily the scenes could be detected under high attentional demands. As a manipulation check, we assessed the identification rates of all the scenes used in the two experiments (63 total) across all five conditions in a different set of participants, to ensure that our color, luminance, and orientation manipulations were in fact influencing the ease of gist extraction.

\section{Method}

Participants A total of 100 participants ( 60 males, 40 females; mean age $=35.26$ years, $18-67$ years old) completed this experiment online on Amazon Mechanical Turk for monetary compensation. Twenty of these participants viewed canonically colored scenes, 20 viewed grayscale scenes, 20 viewed RGB color-inverted scenes, 20 viewed $L^{*} a * b *$ colorinverted scenes, and 20 viewed orientation-inverted scenes. All participants gave online informed consent, approved by the Institutional Review Board of the City University of New York.

Stimuli and procedure The stimuli were presented on participants' personal computers using custom software written in Visual C++ and DirectX libraries. The same scenes (63 total) and random-dot masks used in Experiments 1 and 2 were used as stimuli in this manipulation check. Each trial began with the presentation of a fixation square for 1,000 ms (see Fig. 5). Following this, a scene (subtending an average of $6.02^{\circ} \times$ $4.58^{\circ}$ ) was presented at fixation for $100 \mathrm{~ms}$. A random-dot mask (subtending an average of $19.87^{\circ} \times 19.87^{\circ}$ ) was then presented on the screen for $500 \mathrm{~ms}$. After the mask, the screen went blank, and participants were instructed to identify the scene that had been presented in as few words as possible by typing their description in a textbox on the screen. Each of the 63 scenes was presented once in random order. Each participant viewed only one of the scene sets (canonically colored, grayscale, RGB color-inverted, $L * a * b *$ color-inverted, or orientation-inverted).

\section{Identification results}

Each participant gave one identification response for each scene, yielding 63 responses per participant. Responses were scored blind to experimental condition by two of the authors. Each response was given a score from 0 to 2, depending on how completely the response captured the gist of the image. A score of 2 was given for responses that captured the full gist ${ }^{4}$ of the scene (e.g., "ducks" for a scene featuring ducks in a pond). A score of 1 was given for responses that captured partial gist of the scene (e.g., "water" for the scene featuring ducks in a pond). A score of 0 was given for responses that did not capture the scene gist (e.g., "I don't know"). To measure interrater reliability, Cohen's weighted $\kappa$ (Cohen, 1968) was calculated from the raw scores using the kappa2 function from the R irr package (Gamer, Lemon, Fellows, \& Singh, 2012). Interrater reliability was high, $\kappa_{\mathrm{W}}=.93, p<.001$. The scores for each scene and participant were averaged across raters and divided by the total points available $(2$ points per scene $\times 63$ scenes), to give each participant one average identification score. There was a significant difference in identification performance by scene condition, $F(4,100)=13.54, p<.001, \eta_{\mathrm{p}}^{2}$ $=.36$. Only $18 \%$ (95\% CI [11\%, 25\%]) of the canonically colored scenes were not correctly identified, 39\% (95\% CI $[32 \%, 47 \%]$ ) of the grayscale scenes were not correctly identified, 52\% (95\% CI [45\%, 60\%]) of the RGB color-inverted scenes were not correctly identified, 39\% (95\% CI [32\% $46 \%]$ ) of the $L^{*} a^{*} b^{*}$ color-inverted scenes were not correctly identified, and $46 \%$ (95\% CI [39\%, 54\%]) of the orientationinverted scenes were not correctly identified (see Fig. 6). Planned pairwise comparisons revealed that the identification rate of the canonically colored scenes was significantly higher than the identification rates of the other scene types (all $t \mathrm{~s} \geq$ $5.07, p \mathrm{~s} \leq .001)$. There were no other significant differences between conditions.

\section{Discussion}

We investigated the role of several low-level features - color, luminance, and orientation - in inattentional blindness to realworld scenes and found that color, luminance, and orientation influence the susceptibility of scenes to inattentional blindness under different conditions. In Experiment 1, we investigated inattentional blindness in a serial, dual-task paradigm (high load), and found that the combination of canonical color, canonical luminance, and canonical orientation significantly reduced inattentional blindness relative to canonical luminanceonly information (grayscale scenes), non-canonical color and non-canonical luminance (RGB color-inverted scenes), canonical luminance and non-canonical color $\left(L^{*} a^{*} b *\right.$ color-inverted scenes) and non-canonical orientation. Interestingly, when the load was reduced in Experiment 2 by eliminating the lexical decision task to assess priming, canonical color or canonical luminance alone were sufficient to convey scene content and capture attention.

\footnotetext{
${ }^{4}$ Gist descriptors were obtained by having a separate group of 20 participants describe each of the 63 scenes in a single word. The most common response per scene was used as the gist descriptor for that scene.
} 


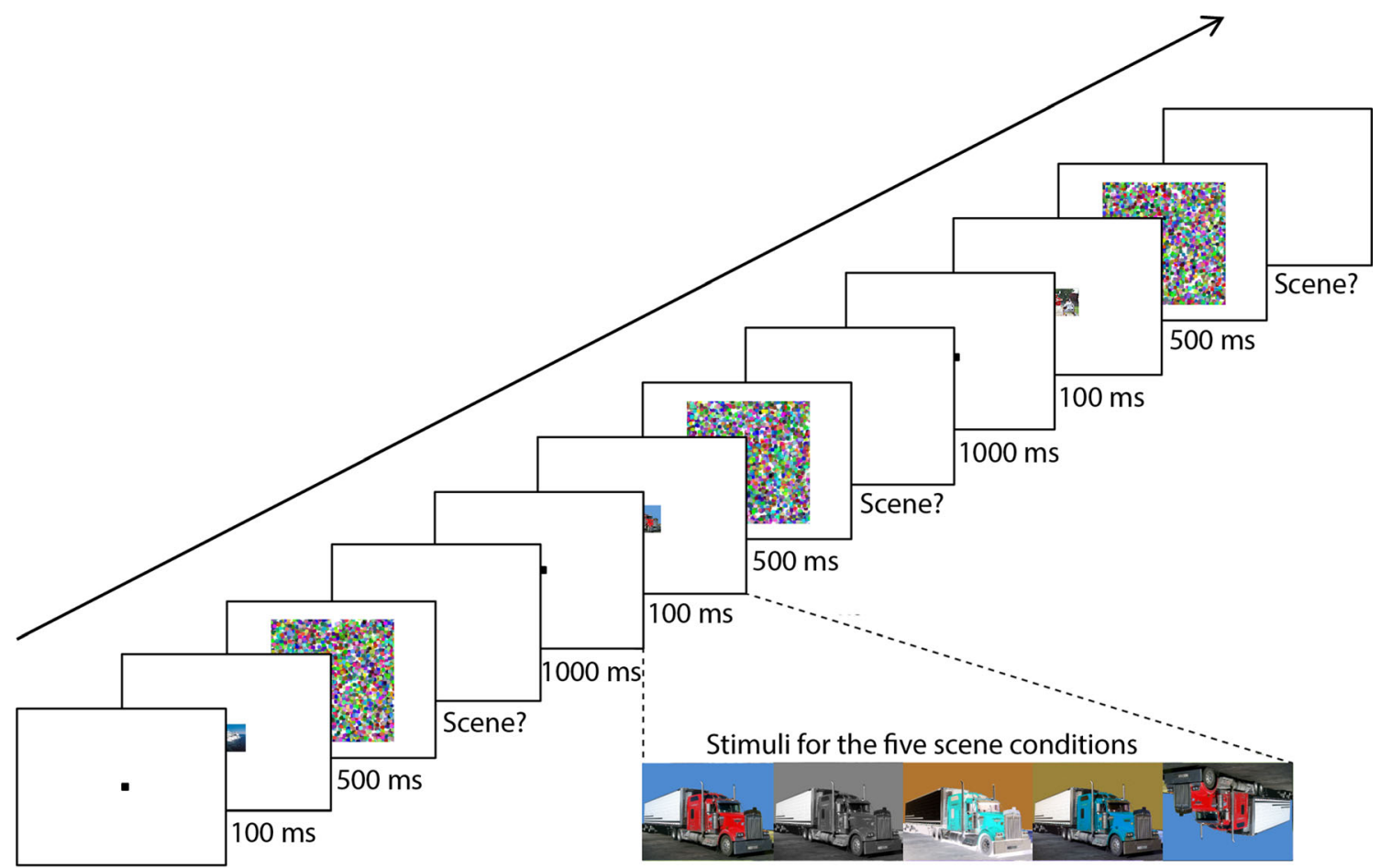

$1000 \mathrm{~ms}$

Fig. 5 Experimental design for the manipulation check. The inset shows example scene stimuli for the canonical color condition, grayscale condition, RGB color-inverted condition, $L^{*} a * b *$ color-inverted condition,

We conducted Experiment 2 to rule out any effects of the priming task on scene awareness in Experiment 1. Presenting words related to the scenes could have triggered scene perception, either through retroperception or priming of later scenes. Furthermore, the repeated presentations of the same scenes

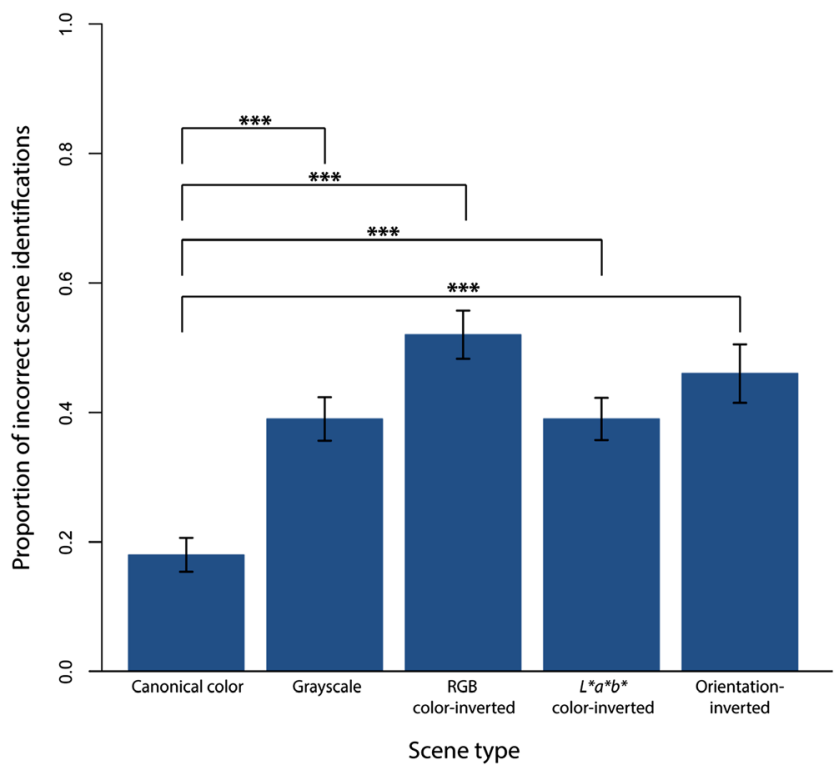

Fig. 6 Proportions of incorrect scene identifications by scene type. Canonically colored scenes were identified correctly significantly more often than any of the other scene types. Error bars reflect $\pm 1 S E$ of the mean. ${ }^{* * *} p<.001$ and orientation-inverted condition, respectively. After the presentation of each scene, participants were asked to identify what was presented

may have aided in the perception and recognition of later presentations of those scenes. In both of these cases, we would expect to see reduced inattentional blindness in Experiment 1 relative to Experiment 2, in which we removed the priming task and used unique scenes. However, we found no significant difference in the rates of inattentional blindness across the two experiments, and the pattern of detection rates across the scene conditions was mostly consistent. This suggests that the inclusion of the priming aspects of Experiment 1 did not increase awareness of the scenes. However, we did detect two interesting differences between the two experiments. In Experiment 2, inattentional blindness to grayscale scenes was significantly reduced relative to rates of inattentional blindness to grayscale scenes in Experiment 1. Furthermore, in Experiment 2, inattentional blindness to the $L^{*} a^{*} b^{*}$ colorinverted scenes did not differ significantly from inattentional blindness to either the canonically colored scenes or the RGB color-inverted scenes. Both of these differences resulted from reduced inattentional blindness in Experiment 2, rather than increased inattentional blindness, which again suggests that the differences were not related to priming of the words on scene perception. Given that both grayscale scenes and $L * a * b *$ color-inverted scenes contain canonical luminance information, these results instead suggest that under certain conditions, canonical luminance also serves as a reliable indicator of scene content that may capture attention and reduce inattentional blindness. 
This likely occurs when load is reduced, as evidenced by the improved performance on the cross task in Experiment 2 , which is discussed in more detail below.

To create conditions of inattention, we used the Mack and Rock cross task (1998) to divert attention away from the scenes. The cross task was intended to be difficult to ensure that it was sufficiently attentionally demanding so as to produce inattentional blindness. The short presentation time $(100 \mathrm{~ms})$ coupled with the randomized cross location meant that in order to perform well, it was important that participants ignored information irrelevant to the cross task. Because the cross task was used to manipulate the focus of attention, we analyzed cross task performance to determine whether differences in rates of inattentional blindness could be explained by differences in allocation of attention. Reduced performance on the cross task could indicate that it was not the focus of attention, which might result in increased awareness of the scenes.

In Experiment 1, performance on the cross task differed significantly across scene conditions. This significant difference was not related to awareness of the scenes, given that there was no significant effect of inattentional blindness status on performance. Rather, the significant difference in performance was driven by reduced performance on the cross task when the scenes were grayscale (see Table 1), regardless of participants' awareness of the scenes. The near-chance performance on the cross task when scenes were grayscale suggests that this condition was especially difficult for participants. This may be because it was the only condition in which both the scenes and the cross were grayscale. In the other conditions, color may have been used to more easily segment the relevant cross information. When both the cross and scenes were grayscale, participants may have had more difficulty identifying the task-relevant information, which impaired their performance.

In Experiment 2, we found no significant differences in cross task performance for any of the variables of interest (critical vs. non-critical trials, inattentionally blind vs. noninattentionally blind participants, and the five experimental conditions). However, performance on the cross task was significantly better in Experiment 2 compared to Experiment 1, suggesting that the task was easier. Because we found no significant differences in rates of inattentional blindness or accuracy on the cross task based on stimulus size in Experiment 2, it is unlikely that performance improved because the stimuli were larger for some participants. Rather, it is more likely that the effect is attentional in nature; when the priming task was removed, the task load was reduced from a dual to a single task. Interestingly, the significantly reduced cross task performance among participants who saw grayscale scenes in Experiment 1 was not detected in Experiment 2, just as the high rates of inattentional blindness to grayscale scenes in Experiment 1 were significantly reduced in Experiment 2. These results suggest that reducing the attentional demands of the experiment disproportionately affected this condition.

Concerns about response biases are discussed frequently in consciousness research but are particularly relevant to studies on inattentional blindness, in which verbal report is often used as the primary measure of awareness. Specifically, there are often concerns about biases for participants to report no awareness to avoid describing uncertain or unclear perceptions. We attempted to control for the bias to report "no" when confidence about perception was low by probing awareness of the scenes multiple times in multiple ways before classifying a participant as inattentionally blind. A participant who is not confident in his or her perception may be inclined to report "no" when asked if he/she noticed anything on the screen aside from the cross and random dot mask. However, when followed up with a more direct question ("Did you notice any scenes?") we anticipated that even unsure participants would report "yes" if they perceived a scene. Finally, if participants said no to both of these questions, they were informed that scenes had been presented during the experiment and were asked to describe any that they could remember. These second and third probes should have spared participants from the discomfort of having to explain precisely what they saw, especially if they were uncertain about their perception. Indeed, several participants answered "no" to the first two probes, but after being told that scenes were present in the experiment, they were able to recall and describe some of the scenes. As a result, we believe this method of assessing scene detection minimized response bias across conditions.

A secondary key finding of this study is that it is possible to produce sustained inattentional blindness to scenes presented multiple times over many trials. ${ }^{5}$ To our knowledge, this is one of the first times that sustained inattentional blindness has been demonstrated using the Mack and Rock (1998) inattentional blindness cross paradigm or a similar paradigm using discrete trials and non-dynamic events (but see Mack \& Clarke 2012, who initially demonstrated that inattentional blindness could be sustained over two critical trials). Numerous studies have demonstrated that inattentional blindness to elements in a video clip can be sustained over time (Becklen \& Cervone, 1983; Neisser, 1979; Neisser \& Becklen, 1975; Simons \& Chabris, 1999; Stoffregen, Baldwin, \& Flynn, 1993). However, these studies examined awareness of a small number of dynamic events embedded within a video presentation. The present study is among the first to show that inattentional blindness can be sustained over many discrete trials by modifying the single critical trial paradigm (see also Ward \& Scholl, 2015, who found what they called

\footnotetext{
${ }^{5}$ It is possible that when a participant reports no awareness at the end of the experiment, the cause is not perceptual, but rather forgetting of the scenes after many trials. However, given the frequent reports of awareness of the scenes across both experiments, we think this explanation is less likely.
} 
"repeated inattentional blindness" for subsequent critical trials after probing on an initial critical trial). The benefit of a single critical trial inattentional blindness paradigm is that it permits investigation of awareness of a single unexpected event. The inherent drawback of this paradigm is that by testing awareness of the unexpected event, the element of inattention is lost; asking participants whether they were aware of something unexpected draws their attention to the critical stimulus in later trials. We have found that it is possible to sustain conditions of inattention by measuring awareness of unexpected stimuli once at the conclusion of many trials. This new paradigm permits investigation of the factors influencing inattentional blindness over time, but prevents precise assessment of when participants become aware of the unexpected stimuli. Each paradigm is well-suited to a different set of questions. This sustained inattentional blindness paradigm may be particularly useful to those seeking to investigate the neural correlates of awareness and the process of becoming aware of unexpected events.

The overall rates of inattentional blindness to scenes in this study may be lower than similar studies as a result of participants having to sustain inattentional blindness over many trials. Our rates of inattentional blindness refer to the percentages of participants who never gained awareness of the scenes over the course of the experiment. These rates are not indicative of the rates of awareness of single scenes presented in a traditional single critical paradigm. In fact, we found high rates of awareness of canonically colored scenes, but using a single critical trial paradigm Cohen et al. (2011) showed the opposite resultthat there is a high rate of inattentional blindness for colored scenes. Taken together, our results suggest that canonical color reduces inattentional blindness to scenes over multiple trials. The reduction of inattentional blindness to repeated presentations of scenes may be the result of several factors. Repeated presentations of the same scenes may have aided in the perception and recognition of later presentations of those scenes. Studies have shown that recognition is more efficient for a subsequent viewing of a stimulus that was perceived and named on first presentation (Bartram, 1974; Biederman \& Cooper, 1991; Schacter et al., 1990). Similarly, Haber and Hershenson (1965) demonstrated in a study of subliminal processing that the probability of perceiving a briefly flashed word increased with the number of presentations. Bar and Biederman (1998) showed that a second presentation of a briefly flashed masked image, occurring 20 trials after the initial presentation, improved naming accuracy of the image from $13.5 \%$ to $34.5 \%$. The stimuli in the Bar and Biederman (1998) study, like those in our study, were presented briefly and masked. It is possible that the repeated presentations of our scenes, even if not consciously perceived, enhanced recognition and perception of later presentations and that as the scenes became easier to recognize, they also became easier to see. Numerous other factors may also contribute to the breakdown of inattentional blindness over time, such as improvement on the central task (freeing attentional resources, which could then be allocated to the scenes) or increased opportunity for momentary failure to attend to the central task.

The masks used in our study may explain some discrepancy between rates of inattentional blindness reported here and elsewhere. Recent research has suggested that scene gist perception is greatly influenced by the choice of mask. Masking functions are strongly affected by mask recognizability and similarity between target and mask spectral characteristics (Hansen \& Loschky, 2013; Loschky, Hansen, Sethi, \& Pydimarri, 2010; Loschky et al., 2009; Loschky et al., 2007). Because we used the same masks that differed only in color across all of our experiments, we do not believe that the masks differentially affected scene gist perception. However, the masks may have interfered with scene gist perception more or less than the masks used in other scene inattentional blindness experiments and therefore should be considered when making comparisons to other studies.

In a third experiment, we measured how color, luminance, and orientation directly affect scene identification. Even though scene gist was identical across all scene condition sets, we found that when scenes contained canonical color, canonical luminance, and canonical orientation information, they were correctly recognized and identified significantly more often than scenes without these canonical features. However, as scene features became less informative of scene content, scene identification was impaired. We found the greatest impairment in identification when scenes were color-inverted in RGB color space. This is likely because RGB color inversion violates both canonical color and canonical luminance relationships. As such, rather than aiding in scene identification, the combination of non-canonical color and non-canonical luminance impede it. Similar, but smaller, impairments in scene identification were noted when scenes were grayscale, $L * a * b *$ color-inverted, or orientation-inverted, suggesting that color, luminance, and orientation information all contribute to the efficient identification of scenes.

Previous work has suggested that inattentional blindness breaks down under conditions that would normally elicit it when critical stimuli are sufficiently meaningful to capture attention (Mack \& Rock, 1998). Stimuli that are more easily recognizable amidst uninterpretable stimuli (frequencyscrambled scenes) may be more meaningful because their gist can be more readily extracted. The scene identification data from our third experiment indicate that scene gist perception is highly efficient when scenes contain canonical color, canonical luminance, and canonical orientation information. Furthermore, these data indicate that when canonical feature relationships are violated, extraction of scene gist is impaired.

Because we used identical paradigms to investigate inattentional blindness across a number of scene conditions, we can speculate about the mechanism through which unattended scenes are brought into conscious awareness. When 
load is high, as it was under the dual-task conditions of Experiment 1, canonical color, canonical luminance, and canonical orientation are all necessary in conjunction to capture attention and release inattentional blindness. Under such conditions, any one of these features alone is less efficient in capturing attention. The reduction of inattentional blindness by canonical features may be because these features are indicative of scene content. This is supported by the scene identification results of Experiment 3, which demonstrated that significantly more scenes were correctly identified when all three canonical features were present than when one or more canonical feature was absent. Under conditions of high load, only highly canonical stimuli were sufficient to capture attention, and it is likely that the combination of these canonical features facilitates the extraction of meaning.

However, under conditions of reduced load (Exp. 2), more attention can be allocated to the scenes, as is evident by the higher performance accuracy on the cross task compared to Experiment 1. With reduced attentional demands, canonical luminance and canonical color alone are sufficient to capture attention. Although the pattern of scene detection results in Experiment 2 does not exactly mirror the rates of correct scene identification in Experiment 3, this is not inconsistent with load moderating the relationship between canonical features and attention capture. Reduction of inattentional blindness requires detection of just one scene, whereas high identification performance requires accurate identification across many scenes. Although non-canonical features may have impaired identification of some scenes, only one salient and meaningful noncanonical scene would still be capable of capturing attention and reducing inattentional blindness. Given that detection rates were higher for most of the conditions in Experiment 2 compared to Experiment 1, differences in detection due to scene identifiability may have been obscured by ceiling effects. However, when non-canonical features are combined (as in the RGB color-inverted scenes, which have both noncanonical color and non-canonical luminance), scenes remained vulnerable to inattentional blindness. Together, these data support the idea that canonical features facilitate the extraction of scene gist, a process that is moderated by attentional demands. We suggest that gist extraction leads to attention capture and ultimately the breakdown of inattentional blindness. However, more research is needed to determine whether it is gist or some other characteristic of canonically colored scenes that captures attention, such as extraction of disjunctive feature sets (Evans \& Treisman, 2005) or individual objects that may not convey scene gist but may still capture attention.

\section{Conclusions}

The experiments presented here provide evidence that canonical color, canonical luminance, and canonical orientation affect the pervasiveness of inattentional blindness to scenes. When attentional load is high, the combination of these features is necessary to extract scene gist and capture attention. However, when attentional demands are reduced, canonical features such as color, luminance, and orientation are sufficient to facilitate gist extraction independently, releasing scenes from inattentional blindness. This pattern of results is consistent with our finding that canonically colored scenes are easiest to identify, whereas grayscale, color-inverted, and orientation-inverted scenes are correctly identified significantly less often. We suggest that the informative value of canonical color, canonical luminance, and canonical orientation may reduce inattentional blindness by facilitating the extraction of scene gist. Stimuli from which the gist can be extracted easily may be more meaningful and capture attention, leading to the breakdown of inattentional blindness.

We also provide evidence that inattentional blindness can be sustained over many trials using a novel paradigm in which the awareness of unexpected stimuli is measured at the conclusion of numerous critical trials. This new paradigm provides opportunities to investigate the factors affecting inattentional blindness over time and the processes contributing to conscious gist perception. This paradigm may be especially useful to those investigating the neural correlates of consciousness and the processes underlying inattentional blindness using fMRI and other imaging techniques, which typically require many trials in order to average out a reliable signal from noise.

Author note This research was supported in part by NSF BCS Grant \#1358893/1561518 and NSF EFRI Grant \#1137172 to T.R.

\section{References}

Bar, M., \& Biederman, I. (1998). Subliminal visual priming. Psychological Science, 9, 464-469. doi:https://doi.org/10.1111/ 1467-9280.00086

Bartram, D. J. (1974). The role of visual and semantic codes in object naming. Cognitive Psychology, 6, 325-356. doi:https://doi.org/10. 1016/0010-0285(74)90016-4

Becklen, R., \& Cervone, D. (1983). Selective looking and the noticing of unexpected events. Memory \& Cognition, 11, 601-608. doi:https:// doi.org/10.3758/BF03197675

Biederman, I., \& Cooper, E. E. (1991). Evidence for complete translational and reflectional invariance in visual object priming. Perception, 20, 585-593. doi:https://doi.org/10.1068/p200585

Biederman, I., \& Ju, G. (1988). Surface versus edge-based determinants of visual recognition. Cognitive Psychology, 20, 38-64.

Biederman, I., Mezzanotte, R. J., \& Rabinowitz, J. C. (1982). Scene perception: Detecting and judging objects undergoing relational violations. Cognitive Psychology, 14, 143-177. doi:https://doi.org/10. 1016/0010-0285(82)90007-X 
Branigan, H. P., Pickering, M. J., \& Cleland, A. A. (1999). Syntactic priming in written production: Evidence for rapid decay. Psychonomic Bulletin \& Review, 6, 635-640.

Castelhano, M. S., \& Henderson, J. M. (2008). The influence of color on the perception of scene gist. Journal of Experimental Psychology: Human Perception and Performance, 34, 660-675. doi:https://doi. org/10.1037/0096-1523.34.3.660

Chaffin, D. B., Andersson, G. B. J., \& Martin, B. J. (1999). Occupational biomechanics (3rd ed.). New York, NY: Wiley.

Clarke, J., Ro, T., \& Mack, A. (2013). The persistence of inattentional blindness and the absence of priming by natural scenes. Journal of Vision, 13(9), 1136. doi:https://doi.org/10.1167/13.9.1136

Cohen, J. (1968). Weighted kappa: Nominal scale agreement provision for scaled disagreement or partial credit. Psychological Bulletin, 70(4), 213-220.

Cohen, M. A., Alvarez, G. A., \& Nakayama, K. (2011). Natural-scene perception requires attention. Psychological Science, 22, 11651172. doi:https://doi.org/10.1177/0956797611419168

Davidoff, J. B., \& Ostergaard, A. L. (1988). The role of colour in categorial judgements. Quarterly Journal of Experimental Psychology, 40A, 533-544.

Delorme, A., Richard, G., \& Fabre-Thorpe, M. (2000). Ultra-rapid categorisation of natural scenes does not rely on colour cues: A study in monkeys and humans. Vision Research, 40, 2187-2200. doi:https://doi.org/10.1016/S0042-6989(00)00083-3

Evans, K. K., \& Treisman, A. (2005). Perception of objects in natural scenes: Is it really attention free? Journal of Experimental Psychology: Human Perception and Performance, 31, 1476-1492. doi:https://doi.org/10.1037/0096-1523.31.6.1476

Fryar, C. D., Gu, Q., Ogden, C. L., \& Flegal, K. M. (2016). Anthropometric reference data for children and adults: United States, 2011-2014 (Vol. 39). Washington, DC: National Center for Health Statistics.

Gamer, M., Lemon, J., Fellows, I., \& Singh, P. (2012). irr: Various coefficients of interrater reliability and agreement. Retrieved from http:// CRAN.R-project.org/package=irr.

Goffaux, V., Jacques, C., Mouraux, A., Oliva, A., Schyns, P., \& Rossion, B. (2005). Diagnostic colours contribute to the early stages of scene categorization: Behavioural and neurophysiological evidence. Visual Cognition, 12, 878-892. doi:https://doi.org/10.1080/ 13506280444000562

Haber, R. N., \& Hershenson, M. (1965). Effects of repeated brief exposures on the growth of a percept. Journal of Experimental Psychology, 69, 40-46. doi:https://doi.org/10.1037/h0021572

Hansen, B. C., \& Loschky, L. C. (2013). The contribution of amplitude and phase spectra-defined scene statistics to the masking of rapid scene categorization. Journal of Vision, 13(13), 21. doi:https://doi. org/10.1167/13.13.21

Intraub, H. (1997). The representation of visual scenes. Trends in Cognitive Sciences, 1, 217-222. doi:https://doi.org/10.1016/ S1364-6613(97)01067-X

Kiefer, M., \& Spitzer, M. (2000). Time course of conscious and unconscious semantic brain activation. NeuroReport, 11, 2401-2407.

Li, F. F., VanRullen, R., Koch, C., \& Perona, P. (2002). Rapid natural scene categorization in the near absence of attention. Proceedings of the National Academy of Sciences, 99, 9596-9601.

Livingstone, M. S., \& Hubel, D. H. (1987). Psychophysical evidence for separate channels for the perception of form, color, movement, and depth. Journal of Neuroscience, 7, 3416-3468.

Loschky, L. C., Hansen, B. C., Sethi, A., \& Pydimarri, T. N. (2010). The role of higher order image statistics in masking scene gist recognition. Attention, Perception, \& Psychophysics, 72, 427-444. doi: https://doi.org/10.3758/APP.72.2.427

Loschky, L. C., Hansen, B., Fintzi, A., Bjerg, A., Ellis, K., Freeman, T., . . Larson, A. (2009). Basic level scene categorization is affected by unrecognizable category-specific image features. Journal of Vision, 9(8), 948. doi:https://doi.org/10.1167/9.8.948

Loschky, L. C., Sethi, A., Simons, D. J., Pydimarri, T. N., Ochs, D., \& Corbeille, J. L. (2007). The importance of information localization in scene gist recognition. Journal of Experimental Psychology: Human Perception and Performance, 33, 1431-1450. doi:https:// doi.org/10.1037/0096-1523.33.6.1431

Mack, A., \& Clarke, J. (2012). Gist perception requires attention. Visual Cognition, 20, 300-327. doi:https://doi.org/10.1080/13506285. 2012.666578

Mack, A., \& Rock, I. (1998). Inattentional blindness. Cambridge, MA: MIT Press.

Neisser, U. (1979). The control of information pickup in selective looking. In A. D. Pick (Ed.), Perception and its development: A tribute to Eleanor J. Gibson (pp. 201-219). Hillsdale, NJ: Erlbaum.

Neisser, U., \& Becklen, R. (1975). Selective looking: Attending to visually specified events. Cognitive Psychology, 7, 480-494. doi:https:// doi.org/10.1016/0010-0285(75)90019-5

Nijboer, T. C. W., Kanai, R., de Haan, E. H. F., \& van der Smagt, M. J. (2008). Recognising the forest, but not the trees: An effect of colour on scene perception and recognition. Consciousness and Cognition, 17, 741-752. doi:https://doi.org/10.1016/j.concog.2007.07.008

Oliva, A., \& Schyns, P. G. (1997). Coarse blobs or fine edges? Evidence that information diagnosticity changes the perception of complex visual stimuli. Cognitive Psychology, 34, 72-107.

Oliva, A., \& Schyns, P. G. (2000). Diagnostic colors mediate scene recognition. Cognitive Psychology, 41, 176-210. doi:https://doi.org/10. 1006/cogp.1999.0728

Potter, M. C. (1975). Meaning in visual search. Science, 187, 965-966. doi:https://doi.org/10.1126/science.1145183

Rousselet, G. A., Fabre-Thorpe, M., \& Thorpe, S. J. (2002). Parallel processing in high-level categorization of natural images. Nature Neuroscience, 5, 629-630. doi:https://doi.org/10.1038/nn866

Sagarin, B. J., Ambler, J. K., \& Lee, E. M. (2014). An ethical approach to peeking at data. Perspectives on Psychological Science, 9, 293-304.

Schacter, D. L., Delaney, S. M., \& Merikle, E. P. (1990). Priming of nonverbal information and the nature of implicit memory. In G. H. Bower (Ed.), The psychology of learning and motivation: Advances in research and theory (Vol. 26, pp. 83-123). San Diego, CA: Academic Press. doi:https://doi.org/10.1016/S0079-7421(08)60052-3

Schyns, P. G., \& Oliva, A. (1994). From blobs to boundary edges: Evidence for time- and spatial-scale-dependent scene recognition. Psychological Science, 5, 195-200. doi:https://doi.org/10.1111/j. 1467-9280.1994.tb00500.x

Sergent, C., Wyart, V., Babo-Rebelo, M., Cohen, L., Naccache, L., \& Tallon-Baudry, C. (2013). Cueing attention after the stimulus is gone can retrospectively trigger conscious perception. Current Biology, 23, 150-155. doi:https://doi.org/10.1016/j.cub.2012.11.047

Shore, D. I., \& Klein, R. M. (2000). The effects of scene inversion on change blindness. Journal of General Psychology, 127, 27-43.

Simons, D. J., \& Chabris, C. F. (1999). Gorillas in our midst: Sustained inattentional blindness for dynamic events. Perception, 28, 10591074. doi:https://doi.org/10.1068/p2952

Stoffregen, T. A., Baldwin, C. A., \& Flynn, S. B. (1993). Noticing of unexpected events by adults with and without mental retardation. American Journal on Mental Retardation, 98, 273-284.

Ward, E. J., \& Scholl, B. J. (2015). Inattentional blindness reflects limitations on perception, not memory: Evidence from repeated failures of awareness. Psychonomic Bulletin \& Review, 22, 722-727. doi: https://doi.org/10.3758/s13423-014-0745-8

Wichmann, F. A., Sharpe, L. T., \& Gegenfurtner, K. R. (2002). The contributions of color to recognition memory for natural scenes. Journal of Experimental Psychology: Learning, Memory, and Cognition, 28, 509-520. doi:https://doi.org/10.1037/0278-7393.28.3.509 